\title{
Positive solutions of a system arising from angiogenesis
}

\author{
Manuel Delgado and Antonio Suárez* \\ Dpto. de Ecuaciones Diferenciales y Análisis Numérico, Fac. de Matemáticas, \\ Univ. de Sevilla, C/. Tarfia s/n, 41012 - Sevilla, SPAIN \\ e-mail: madelgado@us.es,suarez@us.es
}

May 6, 2009

\begin{abstract}
We study a system of equations arising from angiogenesis which contains a nonregular term that vanishes below a certain threshold. This loss of regularity forces one to modify the usual methods of bifurcation theory. Nevertheless, we obtain results on the existence, uniqueness and permanence of a positive solution for the time-dependent problem; and the existence and uniqueness of a positive solution for the stationary one.
\end{abstract}

1991 Mathematics Subject Classification. 35J60, 35K57, 92C17.

Key words. Angiogenesis, bifurcation methods, permanence.

\section{Introduction}

Cancer is a primary area of medical research, and many scientific fields have been involved in the effort to understand and solve this problem. Mathematical modelling can also contribute towards the understanding, description and prediction of the evolution of the process, by offering its own distinct point of view. Such modelling is difficult because cancer is a complicated phenomenon involving many biochemical and physiological processes, which are themselves not completely understood. Given this, deciding which simplifying assumptions are acceptable is a non-trivial task. Furthermore, the models usually present theoretical difficulties, and information about solutions generally has to be obtained via numerical approximations.

${ }^{*}$ The authors acknowledge the support of the MEC under grants BFM2003-06446 and MTM2006-07932. 
This paper considers a mathematical model of angiogenesis, which is an essential phase of the tumor growth (see [13]). It forms part of the process of vascularization of an avascular tumor, which serves to increase the contribution of those nutrients necessary for its growth. This vascularization is also one way to spread the tumor cells to other parts of the body thereby promoting metastasis. Angiogenesis begins with the release of a number of chemical substances (TAF) by the tumor which diffuse into the surrounding tissues, weaken the basal laminas of the endothelial cells (EC) which form the neighboring blood vessels and stimulate the migration of these endothelial cells towards the tumor. In a second step, angiogenesis stimulates the proliferation of the endothelial cells to help the formation of a network of capillary sprouts which vascularizes the tumor.

This second step only begins when TAF concentration reaches a threshold. This fact has been modelled in previous works (for instance, in [2] for an one-dimensional model and [7] for the numerical study of a general model) by means of the introduction of a factor in the equation for the $\mathrm{EC}$ of the form

$$
\mathcal{G}(v)= \begin{cases}0 & \text { if } v \leq v^{*}, \\ v-v^{*} & \text { if } v>v^{*},\end{cases}
$$

where $v^{*}>0$ is a fixed threshold. The presence of this factor, which is not differentiable at $v^{*}$, introduces some mathematical difficulties in the theoretical study of the model and it is therefore interesting initially to try to resolve these difficulties in a very simplified model. This is the first step in the study of a more realistic model in which a chemotaxis term in the EC equation, a third variable modelling the extracellular matrix or even more realistic boundary conditions would be considered.

So, in this paper we are interested in the following system

$$
\begin{cases}u_{t}-\Delta u=\lambda u(1-u) \mathcal{G}(v) & \text { in } \Omega \times(0, T), 0<T \leq \infty, \\ v_{t}-\Delta v=v(\mu-v-c u) & \text { in } \Omega \times(0, T), \\ u=v=0 & \text { on } \partial \Omega \times(0, T), \\ u(0, x)=u_{0}(x)>0, v(0, x)=v_{0}(x)>0 & \text { in } \Omega,\end{cases}
$$

and the corresponding steady-state problem

$$
\begin{cases}-\Delta u=\lambda u(1-u) \mathcal{G}(v) & \text { in } \Omega, \\ -\Delta v=v(\mu-v-c u) & \text { in } \Omega, \\ u=v=0 & \text { on } \partial \Omega\end{cases}
$$

where $\Omega$ is a bounded and regular domain in $\mathbb{R}^{N}, \lambda>0, \mu \in \mathbb{R}, c>0$ and $\mathcal{G}(v)$ is given in (1.1). Here $u$ represents the population density of endothelial cells (EC), and $V$ the concentration of TAF. Following [7], we suppose that mitosis (growth) is governed by a logistic type growth $\lambda u(1-u)$, where $\lambda$ is a positive constant related to the maximum mitotic rate. Mitosis only occurs when the TAF level passes the threshold level $v=v^{*}$. In the second equation, the loss due to the consumption of EC and the balance between the production rate of TAF by the tumor cells and the rate of decay of chemical appear; we model the first one by the term -cuv 
and the second one by the logistic term $\mu v-v^{2}$. This equation means that the concentration of TAF has a logistic growth in the absence of EC. We take Dirichlet boundary conditions.

We will show that the stationary problem (1.3) is a predator-prey type system because there is an a priori bound $u<1$, see Lemma 2.2. In this case it is wellknown that the method of sub- and supersolutions does not give optimal existence results, and for this reason we will study the problem by considering bifurcations with respect to the parameters $\lambda$ and $\mu$. Our main result relating to the existence of solution is:

1. There is no non-negative non-trivial solution of (1.3) for $\mu \leq \lambda_{1}$, where $\lambda_{1}$ is the first eigenvalue of $-\Delta$ with homogeneous Dirichlet conditions; in fact, the only solution is the trivial one.

2. There exists a value $\mu^{*}>\lambda_{1}$ and a function $\lambda_{0}:\left(\mu^{*},+\infty\right) \rightarrow[0,+\infty)$ that is decreasing and satisfies

$$
\lim _{\mu \downarrow \mu^{*}} \lambda_{0}(\mu)=+\infty, \quad \lim _{\mu \uparrow+\infty} \lambda_{0}(\mu)=0
$$

(see Figure 1) such that for $\mu \leq \mu^{*}$ the unique solution of (1.3) is the semitrivial $(0, v)$, and for $\mu>\mu^{*}$ a positive solution of (1.3) exists if and only if $\lambda>\lambda_{0}(\mu)$.

Although the uniqueness of the positive solution for a system like (1.3) is a hard problem (see [5]), we are able to prove that (1.3) has a unique positive solution if $c$ is small. Because of the loss of regularity of the function $\mathcal{G}(v)$ when $v=v^{*}$, we have to use an appropriately adapted version of classical bifurcation results, along with the Implicit Function Theorem.

For the parabolic problem (1.2), we obtain the existence and the uniqueness of a positive solution for all $\lambda>0$ and $\mu \in \mathbb{R}$. We also study the asymptotic behaviour of this solution, showing that if $\mu \leq \lambda_{1}$ then $(u, v) \rightarrow(0,0)$ as $t \rightarrow \infty$; while if $\mu>\lambda_{1}$ and $\lambda<\lambda_{0}(\mu)$ then $(u, v)$ tends to a semitrivial solution of the form $(0, \bar{v})$, and so the $\mathrm{CE}$ concentration tends to zero. Finally, we analyze the interesting case $\lambda>\lambda_{0}(\mu)$. One might hope to find a positive solution of (1.3) that is globally attracting, but this is not an easy task in non order-preserving system. Instead, we prove that the system is permanent, that is, there exists a positive set which is bounded away from zero in each component and which all solutions enter in a finite time. To show this we use an Average Lyapunov Function approach, see Section 4 for details and references.

The organization of the paper is as follows: in Section 2 we study the elliptic system, in Section 3 we analyze the parabolic problem, and we finish with concluding remarks and a biological interpretation of our results. 


\section{Existence and uniqueness of positive solutions of the elliptic system}

Given a regular domain $D \subset \mathbb{R}^{N}$ and $g \in L^{\infty}(D)$ we denote by $\lambda_{1}^{D}(-\Delta+g)$ the first eigenvalue of the problem

$$
-\Delta u+g(x) u=\lambda u \quad \text { in } D, \quad u=0 \quad \text { on } D .
$$

We write $\lambda_{1}^{D}:=\lambda_{1}^{D}(-\Delta)$ and we omit the superscript $D$ when $\Omega=D$.

Now, take a function $g \in L^{\infty}(\Omega), g \geq 0$ and non-trivial. Consider the eigenvalue problem

$$
\begin{cases}-\Delta u=\lambda u g(x) & \text { in } \Omega, \\ u=0 & \text { on } \partial \Omega .\end{cases}
$$

Denote by $\Lambda_{1}(g)$ the first eigenvalue of (2.1). We recall in the following result some of the main properties of $\Lambda_{1}(g)$.

Lemma 2.1 The map $g \in L_{+}^{\infty}(\Omega) \backslash\{0\} \mapsto \Lambda_{1}(g)$ is continuous and decreasing. Moreover, if $g_{n} \in L_{+}^{\infty}(\Omega) \backslash\{0\}$ is such that $\left\|g_{n}\right\|_{\infty} \rightarrow 0$, then

$$
\Lambda_{1}\left(g_{n}\right) \rightarrow+\infty
$$

Proof. Observe that if we denote by

$$
m(\lambda):=\lambda_{1}(-\Delta-\lambda g),
$$

then $m$ is a decreasing map, and $\Lambda_{1}(g)$ is its unique zero. Hence,

$$
m(\lambda)<0 \quad(\text { resp. } m(\lambda)>0) \text { if } \quad \lambda>\Lambda_{1}(g)\left(\operatorname{resp} . \lambda<\Lambda_{1}(g) .\right)
$$

If $f<g$ then $\lambda_{1}(-\Delta-\lambda f)>\lambda_{1}(-\Delta-\lambda g)$, and so $\Lambda_{1}(g)<\Lambda_{1}(f)$. This proves that the map is decreasing. The continuity follows by the continuity of the map $g \mapsto \lambda_{1}(-\Delta-\lambda g)$.

Now, take a sequence $g_{n} \in L_{+}^{\infty}(\Omega) \backslash\{0\}$ such that $\left\|g_{n}\right\|_{\infty} \rightarrow 0$. Then,

$$
0=\lambda_{1}\left(-\Delta-\Lambda_{1}\left(g_{n}\right) g_{n}\right) \geq \lambda_{1}\left(-\Delta-\Lambda_{1}\left(g_{n}\right)\left\|g_{n}\right\|_{\infty}\right)=\lambda_{1}-\Lambda_{1}\left(g_{n}\right)\left\|g_{n}\right\|_{\infty},
$$

and so,

$$
\Lambda_{1}\left(g_{n}\right) \geq \frac{\lambda_{1}}{\left\|g_{n}\right\|_{\infty}}
$$

Hence, $\Lambda_{1}\left(g_{n}\right) \rightarrow+\infty$ as $n \rightarrow \infty$.

Before studying our problem (1.3), consider the logistic equation

$$
\begin{cases}-\Delta u=\sigma u-u^{2} & \text { in } \Omega \\ u=0 & \text { on } \partial \Omega .\end{cases}
$$

It is well-known that (2.3) has a positive solution if, and only if, $\sigma>\lambda_{1}$. Moreover, if there exists a positive solution, the solution is unique and stable; we denote it by 
$\theta_{\sigma}$. We extend $\theta_{\sigma} \equiv 0$ when $\sigma \leq \lambda_{1}$. Furthermore, it is well known that $\theta_{\sigma}<\sigma$, the map $\sigma \in\left(\lambda_{1}, \infty\right) \rightarrow \theta_{\sigma}$ is increasing and $\left\|\theta_{\sigma}\right\|_{\infty} \rightarrow \infty$ as $\sigma \rightarrow \infty$.

It is immediate that any weak solution of (1.3) belongs in fact to $X:=\left[C_{0}^{1}(\bar{\Omega})\right]^{2}$. We have three kinds of solutions of $(1.3)$ : the trivial one $(0,0)$, the semitrivial $\left(0, \theta_{\mu}\right)$ and those with both components non-negative and non-trivial, called coexistence states. If we denote by $\mathcal{K}$ the positive cone of $X$, by the strong maximum principle, any non-negative and non-trivial solution of (1.3) belongs to $\operatorname{int}(\mathcal{K})$.

First, observe that if $(u, v)$ is a solution of (1.3), then $v \leq \theta_{\mu}$, and so if $\mu \leq \lambda_{1}$ we get that $v \equiv 0$, and hence going back to the equation of $u$, we deduce that $u \equiv 0$.

On the other hand, since the map $\mu \mapsto \theta_{\mu}$ is increasing, there exists a value $\mu^{*}>\lambda_{1}$ such that

$$
v^{*}=\max _{\bar{\Omega}} \theta_{\mu^{*}}<\mu^{*} .
$$

Hence if $\mu \leq \mu^{*}$ we get that $\theta_{\mu} \leq \theta_{\mu^{*}} \leq v^{*}$ and so $\mathcal{G}(v) \leq \mathcal{G}\left(\theta_{\mu}\right)=0$; and then $u \equiv 0$. Since we understand completely our problem when $\mu \leq \mu^{*}$, in what follows we assume that

$$
\mu>\mu^{*}>\lambda_{1} .
$$

Our first result provides us a priori bound for the first component of a positive solution of (1.3).

Lemma 2.2 Let $(u, v)$ be a positive solution of (1.3). Then $u<1$ in $\bar{\Omega}$.

Proof. Assume that the set $\Omega_{1}:=\{x \in \Omega: u(x)>1\} \neq \emptyset$. Then,

$$
-\Delta(u-1) \leq 0 \quad \text { in } \Omega_{1}, \quad u-1=0 \quad \text { on } \partial \Omega_{1},
$$

and so, $u \leq 1$ in $\bar{\Omega}_{1}$, which is a contradiction. Therefore, $u \leq 1$ in $\bar{\Omega}$.

Now, take $K$ large enough, then

$$
\begin{cases}(-\Delta+K)(1-u)=(1-u)[K-\lambda u \mathcal{G}(v)] \geq 0 & \text { in } \Omega, \\ 1-u>0 & \text { on } \partial \Omega,\end{cases}
$$

and so, by the strong maximum principle it follows that $u<1$ in $\bar{\Omega}$.

Through this paper, the map

$$
\mu \in\left(\mu^{*},+\infty\right) \mapsto \lambda_{0}(\mu):=\Lambda_{1}\left(\mathcal{G}\left(\theta_{\mu}\right)\right)
$$

plays an important role. In the following result, we show some of its properties.

Proposition 2.1 The map $\mu \mapsto \lambda_{0}(\mu)$ is decreasing, and

$$
\begin{aligned}
& \lim _{\mu \downarrow \mu^{*}} \lambda_{0}(\mu)=+\infty, \\
& \lim _{\mu \uparrow+\infty} \lambda_{0}(\mu)=0 .
\end{aligned}
$$

Proof. Since $\theta_{\mu}$ is increasing in $\mu$, by Lemma 2.1 we conclude the monotony property of $\lambda_{0}(\mu)$ with respect to $\mu$. 
Recall (see (2.2)) that $\lambda_{0}(\mu)$ is the unique root of the map

$$
g(\lambda):=\lambda_{1}\left(-\Delta-\lambda \mathcal{G}\left(\theta_{\mu}\right)\right) .
$$

Since $\theta_{\mu} / \mu \rightarrow 1$ uniformly in compact sets as $\mu \rightarrow+\infty$ (see for instance Theorem 2.1 in [9]), then given an $\varepsilon>0$ and a subdomain $\Omega_{1} \Subset \Omega$ there exists $\mu_{0}>0$ such that for $\mu>\mu_{0}$

$$
v^{*}<\mu(1-\varepsilon) \leq \theta_{\mu} \leq \mu(1+\varepsilon) \quad \text { in } \Omega_{1} .
$$

Then, using the monotonicity of the principal eigenvalue with respect to the domain and the above inequality, we get

$$
\begin{aligned}
& g(\lambda)=\lambda_{1}\left(-\Delta-\lambda \mathcal{G}\left(\theta_{\mu}\right)\right)<\lambda_{1}^{\Omega_{1}}(-\Delta-\lambda \mathcal{G}(\mu(1-\varepsilon)))= \\
& \lambda_{1}^{\Omega_{1}}\left(-\Delta-\lambda\left(\mu(1-\varepsilon)-v^{*}\right)\right)=\lambda_{1}^{\Omega_{1}}-\lambda\left(\mu(1-\varepsilon)-v^{*}\right),
\end{aligned}
$$

and so,

$$
\lambda_{0}(\mu) \leq \frac{\lambda_{1}^{\Omega_{1}}}{\mu(1-\varepsilon)-v^{*}} .
$$

It is easy to conclude that $\lim _{\mu \uparrow+\infty} \lambda_{0}(\mu)=0$.

Finally, since as $\mu \downarrow \mu^{*}$ we get that $\left\|\mathcal{G}\left(\theta_{\mu}\right)\right\|_{\infty} \rightarrow 0$, we can apply again Lemma 2.1 and conclude that $\lambda_{0}(\mu) \rightarrow+\infty$.

We have represented in Figure 1 the curve $\lambda=\lambda_{0}(\mu)$ in the $(\lambda-\mu)$-plane.

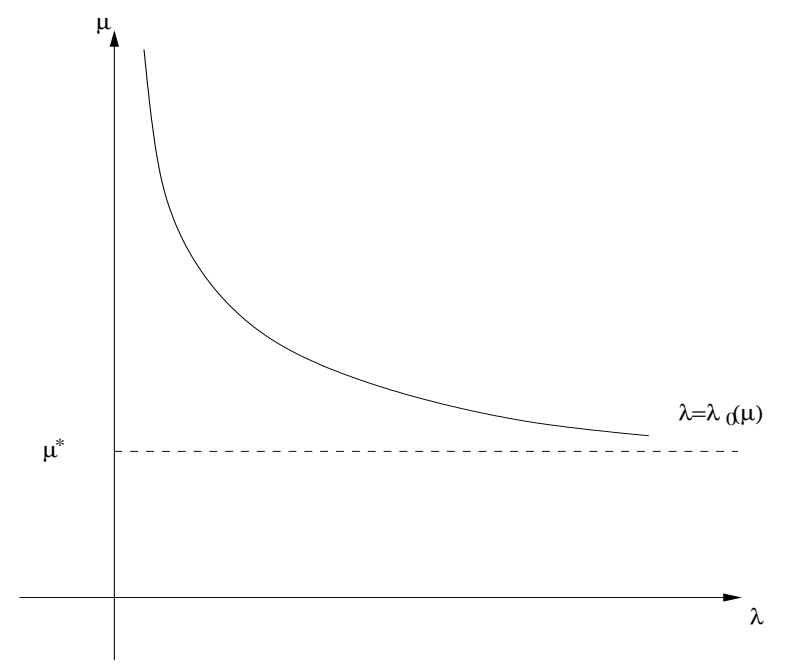

Figure 1: The curve $\lambda=\lambda_{0}(\mu)$.

The study of the following equation will be very useful from now on,

$$
\begin{cases}-\Delta u=\lambda u(1-u) g(x) & \text { in } \Omega, \\ u=0 & \text { on } \partial \Omega .\end{cases}
$$

We get the following existence and uniqueness result. 
Lemma 2.3 Assume $g \in L^{\infty}(\Omega) \backslash\{0\}$, and $\lambda>0$. There exists one positive solution of (2.4) if and only if $\lambda>\Lambda_{1}(g)$. In the case that the positive solution exists, it is unique, we denote it by $v_{[\lambda, g]}$; and it is extended by $v_{[\lambda, g]} \equiv 0$ when $\lambda \leq \Lambda_{1}(g)$.

Moreover,

$$
v_{[\lambda, g]}<1 \quad \text { in } \bar{\Omega}
$$

and if $h \leq g$, then $v_{[\lambda, h]} \leq v_{[\lambda, g]}$.

Proof. First, observe that if $u$ is a positive solution of (2.4) then

$$
0=\lambda_{1}(-\Delta-\lambda(1-u) g(x))>\lambda_{1}(-\Delta-\lambda g),
$$

and so $\lambda>\Lambda_{1}(g)$.

On the other hand, it is not hard to show that $(\underline{u}, \bar{u})=\left(\varepsilon \varphi_{1}, 1\right)$ is a subsupersolution of (2.4) provided that $\varepsilon$ is small enough and $\varphi_{1}$ a positive eigenfunction associated to $\Lambda_{1}(g)$. The uniqueness of positive solution follows by [4]. Estimate (2.5) and the monotonicity with respect to $g$ follow combining the sub-supersolution method and the uniqueness of positive solution.

We are now ready to show the main result about existence:

Theorem 2.1 Assume that $\mu>\mu^{*}$. Then (1.3) possesses at least a positive solution if and only if $\lambda>\lambda_{0}(\mu)$.

Proof. Since $v \leq \theta_{\mu}$, it follows that

$$
0=\lambda_{1}(-\Delta-\lambda(1-u) \mathcal{G}(v))>\lambda_{1}(-\Delta-\lambda \mathcal{G}(v)) \geq \lambda_{1}\left(-\Delta-\lambda \mathcal{G}\left(\theta_{\mu}\right)\right),
$$

and so $\lambda>\lambda_{0}(\mu)$.

In order to prove the existence result we apply the bifurcation method. Observe that the first reaction term in (1.3) is not differentiable, so we will approximate it by regular functions. Indeed, consider the family $\mathcal{G}_{\varepsilon}(s)$ of $C^{1}$ functions, increasing in $s$, decreasing in $\varepsilon, \mathcal{G}_{\varepsilon}(0)=0$ and such that $\mathcal{G}_{\varepsilon} \rightarrow \mathcal{G}$ as $\varepsilon \downarrow 0$ uniformly.

Consider now the approximated problem

$$
\begin{cases}-\Delta u=\lambda u(1-u) \mathcal{G}_{\varepsilon}(v) & \text { in } \Omega, \\ -\Delta v=v(\mu-v-c u) & \text { in } \Omega, \\ u=v=0 & \text { on } \partial \Omega .\end{cases}
$$

Observe that $(0,0)$ is solution of $(2.6)$ and the unique semitrivial solution is $\left(0, \theta_{\mu}\right)$. Denote $\lambda_{\varepsilon}(\mu):=\Lambda_{1}\left(\mathcal{G}_{\varepsilon}\left(\theta_{\mu}\right)\right)$ the principal eigenvalue of

$$
-\Delta u=\lambda u \mathcal{G}_{\varepsilon}\left(\theta_{\mu}\right) \quad \text { in } \Omega, \quad u=0 \quad \text { on } \partial \Omega .
$$

It is clear that

$$
\lambda_{\varepsilon}(\mu) \rightarrow \lambda_{0}(\mu) \quad \text { as } \varepsilon \rightarrow 0 .
$$

We can now apply Theorem 4.1 of [12] and conclude the existence of a continuum (a maximal connected component and closed subset of the set of solutions) $\mathcal{C}_{\varepsilon} \subset$ 
$\mathbb{R} \times \operatorname{int}(\mathcal{K})$ of positive solutions of (2.6) emanating from the semitrivial solution $\left(0, \theta_{\mu}\right)$ at $\lambda=\lambda_{\varepsilon}(\mu)$, such that $\mathcal{C}_{\varepsilon}$ is unbounded in $\mathbb{R} \times \operatorname{int}(\mathcal{K})$.

Now, observe that for any solution $(u, v)$ of $(2.6)$ we have that

$$
u \leq 1 \text { and } v \leq \theta_{\mu} \leq \mu,
$$

and hence $(u, v)$ is bounded in $X$. On the other hand, repeating the arguments used before, we can show that for $\lambda \leq \lambda_{\varepsilon}(\mu)$ the problem (2.6) does not posses any positive solutions.

So, it is clear that $\Pi\left(\mathcal{C}_{\varepsilon}\right)=\left(\lambda_{\varepsilon}(\mu),+\infty\right)$, where $\Pi$ is the projection of $\mathbb{R} \times X$ over $\mathbb{R}$, i. e., $\Pi(\lambda, u)=\lambda$.

Now, we are going to apply the following topological result (see Theorem 9.1 in $[15])$ :

Lemma 2.4 Let $A_{n}$ be a sequence of connected closed sets of a complete metric space $X$ such that $\lim \inf A_{n} \neq \emptyset$, and $\cup_{n} A_{n}$ is relatively compact. Then, $\lim \sup A_{n}$ is non empty, connected and closed.

Consider the metric space $X=\left[C_{0}^{1}(\bar{\Omega})\right]^{2}$ and a sequence $\varepsilon_{n} \rightarrow 0$. Let $\mathcal{C}_{n}$ be the connected component obtained before for $\varepsilon=\varepsilon_{n}$. We are going to show that $\mathcal{C}_{n}$ satisfies the hypotheses of Lemma 2.4. First observe that since $\lambda_{\varepsilon_{n}}(\mu) \rightarrow \lambda_{0}(\mu)$, it is clear that $\lim \inf \mathcal{C}_{n} \neq \emptyset$. Moreover, thanks to the a priori bounds $(2.7), \cup_{n} \mathcal{C}_{n}$ is relatively compact. Indeed, if $\left(\lambda_{k}, u_{k}, v_{k}\right) \in \cup_{n} \mathcal{C}_{n}$ is a sequence bounded in $\mathbb{R} \times X$, then thanks to $(2.7)$ and the elliptic regularity theory we get that $\left(\lambda_{k}, u_{k}, v_{k}\right)$ is bounded in $\mathbb{R} \times\left[W^{2, p}(\Omega)\right]^{2}$ for all $p>1$. Therefore, passing to the limit through a subsequence we get that it is convergent in $\mathbb{R} \times X$.

So, we conclude that $\mathcal{C}:=\lim \sup \mathcal{C}_{n}$ is a connected, closed and nonempty set. Using an standard argument, it can be shown that the elements of $\mathcal{C}$ are solutions of (1.3).

We will prove that $\mathcal{C}$ is non-degenerate, i.e. that $\mathcal{C}$ does not degenerate to the axis $\lambda=\lambda_{0}(\mu)$, or the solutions $\left(0, \theta_{\mu}\right)$ or $(0,0)$.

First, observe that at $\lambda=\lambda_{0}(\mu)$ the continuum can not degenerate because (1.3) does not have solution for $\lambda=\lambda_{0}(\mu)$.

Now, assume that for a sequence of solutions $\left(\lambda_{n}, u_{n}, v_{n}\right) \in \mathcal{C}_{n}$ we have that $\left(\lambda_{n}, u_{n}, v_{n}\right) \rightarrow(\bar{\lambda}, 0,0)$, with $\bar{\lambda}>\lambda_{0}(\mu)$. Define

$$
U_{n}=\frac{u_{n}}{\left\|u_{n}\right\|_{\infty}} .
$$

Then, it is clear that

$$
-\Delta U_{n}=\lambda_{n} U_{n}\left(1-u_{n}\right) \mathcal{G}_{\varepsilon_{n}}\left(v_{n}\right),
$$

and so $-\Delta U=0$ in $\Omega, U=0$ on $\partial \Omega$, and $\|U\|_{\infty}=1$, an absurdum.

Analogously, if $\left(\lambda_{n}, u_{n}, v_{n}\right) \in \mathcal{C}_{n}$ is such that $\left(\lambda_{n}, u_{n}, v_{n}\right) \rightarrow\left(\bar{\lambda}, 0, \theta_{\mu}\right)$ with $\bar{\lambda}>$ $\lambda_{0}(\mu)$, then

$$
-\Delta U=\bar{\lambda} U \mathcal{G}\left(\theta_{\mu}\right),
$$

and so $\bar{\lambda}=\lambda_{0}(\mu)$, again an absurdum. 


\subsection{The uniqueness result}

Before proving the uniqueness result, we analyze the behaviour of (1.3) near to $c=0$. It is clear that if $\lambda>\lambda_{0}(\mu)$ and $\mu>\lambda_{1}$, (1.3) possesses a unique positive solution $\left(u_{0}, v_{0}\right)$ for $c=0$, and it verifies

$$
\begin{cases}-\Delta u_{0}=\lambda u_{0}\left(1-u_{0}\right) \mathcal{G}\left(v_{0}\right) & \text { in } \Omega, \\ -\Delta v_{0}=v_{0}\left(\mu-v_{0}\right) & \text { in } \Omega, \\ u_{0}=v_{0}=0 & \text { on } \partial \Omega .\end{cases}
$$

Observe that $v_{0}=\theta_{\mu}$ and $u_{0}=v_{\left[\lambda, \mathcal{G}\left(v_{0}\right)\right]}$. The following result shows that the solutions of $(1.3)$ are near to $\left(u_{0}, v_{0}\right)$ when $c$ is small. Its proof is similar to, for instance, Lemma 3.1 in [3].

Lemma 2.5 Let $\left(u_{c}, v_{c}\right)$ be any positive solution of (1.3). Then,

$$
\left(u_{c}, v_{c}\right) \rightarrow\left(u_{0}, v_{0}\right) \quad \text { uniformly as } c \downarrow 0 .
$$

Theorem 2.2 Fix $\lambda>0$ and $\mu>\mu^{*}$ such that $\lambda>\lambda_{0}(\mu)$. There exists $c_{0}=$ $c_{0}(\lambda, \mu)>0$ such that for $c<c_{0}$ there exists a unique coexistence state of (1.3).

Proof. The proof is based on the Implicit Function Theorem. Observe that the nonlinearities of our systems are non-differentiable, so we can not apply directly the mentioned result.

Denote by $L:=(-\Delta)^{-1}$, subject to homogeneous Dirichlet condition, and $Y:=$ $\left[W^{2, p}(\Omega) \cap W_{0}^{1, p}(\Omega)\right]^{2}$ with $p>N$. Define the map $F: \mathbb{R} \times X \mapsto Y$ by

$$
F(c, u, v):=\left(u-\lambda L[u(1-u) \mathcal{G}(v)], v-L\left[\mu v-v^{2}-c u v\right]\right) .
$$

Thanks to the elliptic regularity, $F$ is well defined. Also, it is clear that $(u, v)$ is solution of (1.3) if, and only if, $F(c, u, v)=0$. And, finally $F\left(0, u_{0}, v_{0}\right)=0$. We are going to apply the Implicit Function Theorem in [8], see the remark after Theorem 15.1 in [8]. For that, we need to find a homeomorphism $\mathcal{L} \in L(X, Y)$ and a constant $k>0$ such that

$$
\left\|F(c, u, v)-F(c, \bar{u}, \bar{v})-\mathcal{L}(u-\bar{u}, v-\bar{v})^{t}\right\|_{Y} \leq k\left\|(u-\bar{u}, v-\bar{v})^{t}\right\|_{X},
$$

for $(u, v),(\bar{u}, \bar{v}) \in B_{X}\left(\left(u_{0}, v_{0}\right), r_{0}\right)$ and $c<c_{0}$ for some $c_{0}>0$ and $r_{0}>0$ to determine, and $k$ such that $k\left\|\mathcal{L}^{-1}\right\|_{L(Y, X)}<1$, where $B_{X}\left(\left(u_{0}, v_{0}\right), r_{0}\right)$ denotes the ball in $X$ centered at $\left(u_{0}, v_{0}\right)$ with radius $r_{0}$.

Now, we build $\mathcal{L}$. Observe that the nonlinear part of the first component of $F(c, u, v)-F(c, \bar{u}, \bar{v})$ can be written as

$$
\begin{gathered}
u(1-u) \mathcal{G}(v)-\bar{u}(1-\bar{u}) \mathcal{G}(\bar{v})=(u(1-u)-\bar{u}(1-\bar{u})) \mathcal{G}(v)+\bar{u}(1-\bar{u})(\mathcal{G}(v)-\mathcal{G}(\bar{v}))= \\
=\left[\left(1-2 u_{0}\right) \mathcal{G}\left(v_{0}\right)-\left(1-2 u_{0}\right) \mathcal{G}\left(v_{0}\right)+(1-u-\bar{u}) \mathcal{G}(v)\right](u-\bar{u})+ \\
{\left[\bar{u}(1-\bar{u})\left(\frac{\mathcal{G}(v)-\mathcal{G}(\bar{v})}{v-\bar{v}}\right)-\chi(x) u_{0}\left(1-u_{0}\right)+\chi(x) u_{0}\left(1-u_{0}\right)\right](v-\bar{v}),}
\end{gathered}
$$


where

$$
\chi(x):=\chi_{\left\{\theta_{\mu}>v^{*}\right\}},
$$

$\left(\chi_{A}\right.$ stands for the characteristic function of a set $A \subset \mathbb{R}^{N}$ ). With a similar reasoning to the second equation, we can show that

$$
F(c, u, v)-F(c, \bar{u}, \bar{v})=\mathcal{L}(u-\bar{u}, v-\bar{v})^{t}+\mathcal{K}(u-\bar{u}, v-\bar{v})^{t},
$$

where $\mathcal{L}=\left(L_{i j}\right), \mathcal{K}=\left(K_{i j}\right)$ with

$$
\begin{aligned}
& L_{11} \xi:=\xi-\lambda L\left[\left(1-2 u_{0}\right) \mathcal{G}\left(v_{0}\right) \xi\right], \\
& L_{12} \eta:=-\lambda L\left[\chi(x) u_{0}\left(1-u_{0}\right) \eta\right],
\end{aligned}
$$$$
L_{21}:=0
$$$$
L_{22} \eta:=\eta-L\left[\left(\mu-2 v_{0}\right) \eta\right] \text {, }
$$$$
K_{11} \xi:=L[A(x) \xi], \quad K_{12} \eta:=L[B(x) \eta], \quad K_{21} \xi:=L[C(x) \xi], \quad K_{22} \eta:=L[D(x) \eta],
$$

where

$$
\begin{aligned}
& A(x):=-\lambda\left(\mathcal{G}(v)(1-u-\bar{u})-\mathcal{G}\left(v_{0}\right)\left(1-2 u_{0}\right)\right), \\
& B(x):=-\lambda\left(\bar{u}(1-\bar{u})\left(\frac{\mathcal{G}(v)-\mathcal{G}(\bar{v})}{v-\bar{v}}\right)-\chi(x) u_{0}\left(1-u_{0}\right)\right), \\
& C(x):=c \bar{v} \\
& D(x):=-\left(2 v_{0}-v-\bar{v}-c u\right) .
\end{aligned}
$$

First, we show that $\mathcal{L}^{-1}$ is well-defined. Take $(f, g)^{t} \in Y$, we have to prove that there exists a unique $(\xi, \eta) \in X$ such that

$$
\mathcal{L}(\xi, \eta)^{t}=(f, g)^{t},
$$

or equivalently,

$$
\begin{cases}\left(-\Delta-\lambda \mathcal{G}\left(v_{0}\right)\left(1-2 u_{0}\right)\right) \xi-\lambda u_{0}\left(1-u_{0}\right) \chi(x) \eta=-\Delta f & \text { in } \Omega, \\ \left(-\Delta-\left(\mu-2 v_{0}\right)\right) \eta=-\Delta g & \text { in } \Omega, \\ \xi=\eta=0 & \text { on } \partial \Omega .\end{cases}
$$

Observe that since $v_{0}$ is a positive solution of $-\Delta v_{0}=v_{0}\left(\mu-v_{0}\right)$, we get

$$
\lambda_{1}\left(-\Delta-\left(\mu-2 v_{0}\right)\right)>\lambda_{1}\left(-\Delta-\left(\mu-v_{0}\right)\right)=0,
$$

and so $\eta$ is well defined. On the other hand, since $u_{0}$ is a positive solution of $-\Delta u_{0}=\lambda u_{0}\left(1-u_{0}\right) \mathcal{G}\left(v_{0}\right)$, we get

$$
0=\lambda_{1}\left(-\Delta-\lambda\left(1-u_{0}\right) \mathcal{G}\left(v_{0}\right)\right)<\lambda_{1}\left(-\Delta-\lambda \mathcal{G}\left(v_{0}\right)\left(1-2 u_{0}\right)\right),
$$

and so $\xi$ is also well-defined.

Hence,

$$
\begin{aligned}
\left\|F(c, u, v)-F(c, \bar{u}, \bar{v})-\mathcal{L}(u-\bar{u}, v-\bar{v})^{t}\right\|_{Y}= & \left\|\mathcal{K}(u-\bar{u}, v-\bar{v})^{t}\right\|_{Y} \\
& \leq\|\mathcal{K}\|_{L(X, Y)}\left\|(u-\bar{u}, v-\bar{v})^{t}\right\|_{X} .
\end{aligned}
$$


Now, we compute $\|\mathcal{K}\|_{L(X, Y)}$. Take $\left\|(\xi, \eta)^{t}\right\|_{X}=1$ and denote by $(f, g)^{t}=\mathcal{K}(\xi, \eta)^{t}$, then

$$
\left\{\begin{array} { l l } 
{ - \Delta f = A ( x ) \xi + B ( x ) \eta } & { \text { in } \Omega , } \\
{ f = 0 } & { \text { on } \partial \Omega , }
\end{array} \quad \left\{\begin{array}{ll}
-\Delta g=C(x) \xi+D(x) \eta & \text { in } \Omega, \\
g=0 & \text { on } \partial \Omega
\end{array}\right.\right.
$$

and so by the elliptic regularity

$$
\|f\|_{2, p} \leq K\left\{\|A \xi\|_{p}+\|B \eta\|_{p}\right\}, \quad\|g\|_{2, p} \leq K\left\{\|C \xi\|_{p}+\|D \eta\|_{p}\right\},
$$

for some positive constant $K$. Now, by Lemma 2.5 we have that $A(x) \rightarrow 0$ pointwise as $c \rightarrow 0$, and similarly to the functions $C$ and $D$. So, using the dominated convergence theorem, we get that

$$
\|A\|_{p},\|C\|_{p},\|D\|_{p} \rightarrow 0 \quad \text { as } c \rightarrow 0 .
$$

We need to show a similar result to $B$. Denoting by

$$
G_{c}(x):=\frac{\mathcal{G}(v)(x)-\mathcal{G}(\bar{v})(x)}{v(x)-\bar{v}(x)},
$$

we can show that

$$
\left|G_{c}\right| \leq 1
$$

and that pointwise

$$
G_{c}(x) \rightarrow \chi(x) .
$$

Then, the dominated convergence theorem concludes that $\|B\|_{p} \rightarrow 0$ as $c \rightarrow 0$. Therefore,

$$
\|\mathcal{K}\|_{L(X, Y)}=\sup _{\left\|(\xi, \eta)^{t}\right\|_{X}=1}\left\|\mathcal{K}(\xi, \eta)^{t}\right\|_{Y} \rightarrow 0 \quad \text { as } c \rightarrow 0 .
$$

Hence, there exists $c_{0}>0$ such that for $c<c_{0}$ we have that

$$
\|\mathcal{K}\|_{L(X, Y)} \leq k
$$

for $k$ such that $k\left\|\mathcal{L}^{-1}\right\|_{L(Y, X)}<1$. This proves (2.8) and completes the proof.

\section{The time dependent problem}

We denote by $\Theta_{\sigma}$ the unique positive solution of

$$
\begin{cases}u_{t}-\Delta u=\sigma u-u^{2} & \text { in } \Omega \times(0, T), T \leq \infty, \\ u=0 & \text { on } \partial \Omega \times(0, T), \\ u(0, x)=u_{0}(x)>0 & \text { in } \Omega\end{cases}
$$

It holds (see Theorem 4.4 in [5]) that

$$
\Theta_{\sigma} \rightarrow \theta_{\sigma} \quad \text { in } C^{1}(\bar{\Omega}) \text { as } t \rightarrow+\infty .
$$


Before studying the system (1.2), we analyze the following equation

$$
\begin{cases}u_{t}-\Delta u=\lambda u(1-u) G(x, t) & \text { in } \Omega \times(0, T), \\ u=0 & \text { on } \partial \Omega \times(0, T), \\ u(0, x)=u_{0}(x)>0, & \text { in } \Omega\end{cases}
$$

where $G \in C^{\gamma}\left(\bar{\Omega} \times \mathbb{R}_{+}\right)$for some $\gamma \in(0,1), G \geq 0$ and non-trivial, and for instance $u_{0} \in C_{0}^{1}(\bar{\Omega})$. The following result shows the existence and uniqueness of positive solution of (3.2) and its asymptotic behaviour. Its proof is standard, see for instance [14] and [5].

Proposition 3.1 1. There exists a unique positive solution of (3.2), denoted by $\Upsilon_{[\lambda, G]}$.

2. Assume that $g \in C^{\gamma}(\bar{\Omega}), \gamma \in(0,1), g \geq 0$ and non-trivial. Then,

$$
\Upsilon_{[\lambda, g]} \rightarrow v_{[\lambda, g]}, \quad \text { in } C^{1}(\bar{\Omega}) \text { as } t \rightarrow+\infty .
$$

where $v_{[\lambda, g]}=0$ when $\lambda \leq \Lambda_{1}(g)$.

The following result shows the existence and uniqueness of positive solution for $(1.2)$.

Proposition 3.2 Take $\left(u_{0}, v_{0}\right) \in X, u_{0}, v_{0}>0$. There exists a unique positive solution $(u, v) \in\left(C(\bar{\Omega} \times[0, T]) \cap C^{1,2}(\Omega \times(0, T))\right)^{2}$ of $(1.2)$.

Proof. Observe that in this case we can not assure that $u<1$ because we do not know the size of $u_{0}$. So, our coupled system (1.2) has non-monotone nonlinearities. Anyway, we can apply the general sub-supersolution method (see the definition 8.9.1 in [14] for instance) with

$$
(\underline{u}, \bar{u})=(0, K), \quad(\underline{v}, \bar{v})=\left(0, \Theta_{\mu}\right),
$$

where $K$ is a positive constant such that $K \geq \max \left\{1,\left\|u_{0}\right\|_{\infty}\right\}$. Observe that although the nonlinearities are not $C^{1}$, they are Lipschitz and satisfy the condition (8.8.15) in [14], and so we can apply the method, see Theorem 8.9.3 in [14].

The positivity and uniqueness follow easily.

With respect to the asymptotic behavior, we get

Proposition 3.3 Assume that $\lambda<\lambda_{0}(\mu)$. Then if $(u, v)$ is the positive solution of (1.2), we have that

$$
(u, v) \rightarrow\left(0, \theta_{\mu}\right) \quad \text { as } t \rightarrow+\infty
$$

where $\theta_{\mu}=0$ if $\mu \leq \lambda_{1}$.

Proof. First consider $\mu \leq \lambda_{1}$. Observe that if $(u, v)$ is the solution of (1.2), then

$$
v \leq \Theta_{\mu} .
$$


So, $v \rightarrow 0$ as $t \rightarrow \infty$. Hence, for $t$ large we get that $v \leq v^{*}$, and going back to the equation for $u$, we deduce that $u \rightarrow 0$ as $t \rightarrow \infty$.

Second, assume that $\lambda_{1}<\mu<\mu^{*}$. Take $\varepsilon>0$ positive and small such that

$$
\left\|\theta_{\mu}\right\|_{\infty}+\varepsilon<\left\|\theta_{\mu^{*}}\right\|_{\infty}=v^{*}
$$

So, using (3.3) for large $t$ we have that

$$
v \leq \theta_{\mu}+\varepsilon<v^{*}
$$

and then $u \rightarrow 0$ and we conclude that $v \rightarrow \theta_{\mu}$ as $t \rightarrow \infty$.

Now assume that $\mu>\mu^{*}>\lambda_{1}$ and $\lambda<\lambda_{0}(\mu)=\Lambda_{1}\left(\mathcal{G}\left(\theta_{\mu}\right)\right)$. By the continuity of $\Lambda_{1}(H)$ with respect to the function $H$, there exists $\varepsilon>0$ such that

$$
\lambda<\Lambda_{1}\left(\mathcal{G}\left(\theta_{\mu}+\varepsilon\right)\right) .
$$

Observe that again by (3.3), there exists $t_{0}>0$, such that for $t \geq t_{0}$, we get

$$
v \leq \theta_{\mu}+\varepsilon
$$

So, for $t \geq t_{0}$

$$
u_{t}-\Delta u=\lambda u(1-u) \mathcal{G}(v) \leq \lambda u \mathcal{G}(v) \leq \lambda u \mathcal{G}\left(\theta_{\mu}+\varepsilon\right)
$$

Hence, $u$ is a subsolution of the linear problem

$$
z_{t}-\Delta z=\lambda z \mathcal{G}\left(\theta_{\mu}+\varepsilon\right)
$$

and since $\lambda<\Lambda_{1}\left(\mathcal{G}\left(\theta_{\mu}+\varepsilon\right)\right)$ we get $z \rightarrow 0$, and so it follows that

$$
u \rightarrow 0 \quad \text { as } t \rightarrow+\infty \text {. }
$$

Hence, we conclude that $u \rightarrow 0$, and then $v \rightarrow \theta_{\mu}$ as $t \rightarrow \infty$.

Finally, we consider the case $\mu=\mu^{*}$. Fixed $\lambda>0$, thanks to Lemma 2.1 there exists $\varepsilon>0$ small enough such that

$$
\lambda<\Lambda_{1}\left(\mathcal{G}\left(\theta_{\mu^{*}}+\varepsilon\right)\right) .
$$

Now, we can reason similarly to the above case.

Now, we want to show that for $\lambda>\lambda_{0}(\mu)$ the solution of of (1.2) enters and remains in a bounded set of $X$ for away from zero for large time, that is the system is permanent. For that, we use a similar argument to [6], see also [5].

In order to formalize this, we need some notation. Define the map

$$
\pi: \mathcal{K} \times[0, \infty) \mapsto \mathcal{K}, \quad \pi\left(u_{0}, v_{0}, t\right):=(u, v)
$$

being $(u, v)$ the unique solution of (1.2) in $t$ initially in $\left(u_{0}, v_{0}\right)$. We will show some properties of $\pi$ later. We say that (1.2) is permanent if there exists a bounded set $U \subset X$ such that 
1. $\inf _{u \in U} d(u, \partial \mathcal{K})>0$, where $d(u, \partial \mathcal{K})=\inf _{v \in \partial \mathcal{K}} d(u, v)$, and

2. $\lim _{t \rightarrow \infty} d\left(\pi\left(\left(u_{0}, v_{0}\right), t\right), U\right)=0$ for all $\left(u_{0}, v_{0}\right) \in \operatorname{int}(\mathcal{K})$.

Our main result reads:

Theorem 3.1 Assume that $\lambda>\lambda_{0}(\mu)$. Then the system (1.2) is permanent.

For that, we use the following result (see Corollary 2.3 of [11] or Theorems 4.1 and 4.2 in [5]).

Theorem 3.2 Assume that $\pi$ is dissipative and $\pi(\cdot, t)$ is compact for $t \geq t_{0}$ for some $t_{0}>0$. Let $\mathcal{A}$ denote the global attractor for $\pi$ and

$$
X^{\prime}:=\overline{\pi\left(B(\mathcal{A}, \varepsilon),\left[t_{0}, \infty\right)\right)} \quad \text { and } \quad X^{\prime \prime}:=\pi\left(X^{\prime}, t^{\prime}\right)
$$

for some $t^{\prime}$ large, and $B(\mathcal{A}, \varepsilon)$ is a neighbourhood of $\mathcal{A}$. Finally, denote by

$$
S:=X^{\prime \prime} \cap \partial \mathcal{K} \text {. }
$$

Assume that there exists a continuous function $P: X^{\prime \prime} \mapsto[0,+\infty)$ with $P(u, v)=0$ if and only if $(u, v) \in S$, and define

$$
a(t,(u, v))=\liminf _{\left(u_{0}, v_{0}\right) \rightarrow(u, v)}\left(\frac{P\left(\pi\left(u_{0}, v_{0}, t\right)\right)}{P\left(u_{0}, v_{0}\right)}\right), \quad \text { with }\left(u_{0}, v_{0}\right) \in X^{\prime \prime} \backslash S .
$$

Then, (1.2) is permanent if

$$
\sup _{t>0} a(t,(u, v))> \begin{cases}1 & (u, v) \in \omega(S) \\ 0 & (u, v) \in S\end{cases}
$$

where $\omega(S)$ denotes the $\omega$-limit set.

We need to check the hypotheses of the above theorem.

Lemma 3.1 The map $\pi$ is well defined, it is dissipative and compact for large $t$.

Proof. First, we show that the solutions are bounded in $L^{\infty}$. Observe that since $v \leq \Theta_{\mu}$ and $\Theta_{\mu} \rightarrow \theta_{\mu}<\mu$ as $t \rightarrow \infty$, there exists $t_{0}=t\left(v_{0}\right)$ such that for $t \geq t_{0}$

$$
v \leq \Theta_{\mu} \leq \mu+1 \text {. }
$$

Denote now by $z\left(t ; z_{0}\right)$ the unique positive solution of

$$
\begin{cases}z_{t}-\Delta z=\lambda z(1-z) \chi_{\{z<1\}} \mathcal{G}(\mu+1) & \text { in } \Omega \times(0,+\infty), \\ z=0 & \text { on } \partial \Omega \times(0,+\infty), \\ z(0, x)=z_{0}(x)>0 & \text { in } \Omega\end{cases}
$$

It is not hard to show that there exists a unique positive solution $z$ of (3.5). Moreover, the steady state equation to (3.5) is similar to (2.4) with $g=\mathcal{G}(\mu+1)$, and hence by $(2.5)$

$$
z \rightarrow v_{[\lambda, \mathcal{G}(\mu+1)]}<1, \quad \text { as } t \rightarrow+\infty .
$$


Thus, for $t \geq t_{1}\left(z_{0}\right)$ we get

$$
z \leq 1
$$

Now, take $t \geq t_{0}$ and consider $\bar{z}$ the solution of (3.5) with $z(0, x)=u\left(t_{0}, x\right)$. It is clear that since $v \leq \mu+1, \bar{z}$ is supersolution of the $u$-equation and so

$$
u \leq \bar{z} \leq 1
$$

for $t \geq t_{1}=t\left(u_{0}\right)$. Thus, for $t \geq t_{2}:=\max \left\{t_{0}, t_{1}\right\}$ there exist $M_{i}, i=1,2$ such that

$$
u(x, t) \leq M_{1}, \quad v(x, t) \leq M_{2} .
$$

Now, denote by

$$
E:=\left[C_{0}^{0}(\bar{\Omega})\right]^{2}
$$

and $h_{i}:[0, \infty) \rightarrow C_{0}^{0}(\bar{\Omega})$ as

$$
h_{1}(r):=\lambda u(x, r)(1-u(x, r)) \mathcal{G}(v(x, r)), \quad h_{2}(r):=v(x, r)(\mu-v(x, r)-c u(x, r)) .
$$

Thanks to the bound (3.6), we have that $\sup _{r \in[0, \infty)}\left\|h_{i}(r)\right\|_{C_{0}^{0}(\bar{\Omega})}<\infty$, and by Lemma 3.2 in [6] we get hat $(u(t), v(t)) \in E^{\beta}$, where $E^{\beta}$ denotes the fractional power space, $\beta \in(0,1)$ and given $\bar{t}>0$, there exist $M_{i}(\bar{t}), i=1,2$, depending only on $\bar{t}$ such that for $t \geq \bar{t}$

$$
\|(u, v)\|_{E^{\beta}} \leq M_{1}(\bar{t})\left\|\left(u_{0}, v_{0}\right)\right\|_{E^{0}}+M_{2}(\bar{t}) \sup _{r \in[0, \infty)}\left\|\left(h_{1}(r), h_{2}(r)\right)\right\|_{E^{0}} .
$$

Taking $q=1$ in Lemma 3.1 of [6], getting $E^{\beta} \hookrightarrow\left[C_{0}^{1}(\bar{\Omega})\right]^{2}$ for $\beta>1 / 2$. So,

$$
\|(u, v)\|_{\left[C^{1}(\bar{\Omega})\right]^{2}} \leq M(\bar{t})
$$

and therefore $\pi$ is well defined and it is dissipative in $\mathcal{K}$.

We will show now the compactness of $\pi$. Now, take $\left(u_{0}, v_{0}\right) \in\left[C_{0}^{1}(\bar{\Omega})\right]^{2}$, and so $u_{0} \in E^{0}:=L^{q}(\Omega)$ for all $q \geq 1$. It is clear that $h_{1}(r) \in L^{q}(\Omega)$ for all $q \geq 1$ and $\left\|h_{1}(r)\right\|_{q} \leq C$. Putting $A=-\Delta$, we can write the solution $u$ as

$$
u(t)=e^{-A t} u_{0}+\int_{0}^{t} e^{-A(t-s)} h_{1}(s) d s .
$$

Consider the complex interpolation spaces $E^{\beta}$ between $E^{0}=L^{q}(\Omega)$ and $E^{1}:=$ $W^{2, q}(\Omega) \cap W_{0}^{1, q}(\Omega)$. It is known that $E^{\beta}=L^{2 \beta, q}(\Omega)$ are the Bessel potential spaces, see [1]. Hence,

$$
\|u(t)\|_{\beta}=\left\|A^{\beta} u(t)\right\|_{q} \leq\left\|e^{-A t} u_{0}\right\|_{\beta}+\int_{0}^{t}\left\|e^{-A(t-s)} h_{1}(s)\right\|_{\beta} d s .
$$

On the other hand, by Theorem 1.4.3 in [10] we have

$$
\left\|e^{-A t} u_{0}\right\|_{\beta} \leq C_{\beta} t^{-\beta} e^{-\delta t}\left\|u_{0}\right\|_{q}
$$


for some $\delta>0$ and $C_{\beta}>0$. Then,

$$
\|u(t)\|_{\beta} \leq C_{\beta}\left[t^{-\beta} e^{-\delta t}\left\|u_{0}\right\|_{q}+\sup _{s \in[0, t]}\left\|h_{1}(s)\right\|_{q} \int_{0}^{t} \frac{e^{-\delta(t-s)}}{(t-s)^{\beta}} d s\right],
$$

and so,

$$
\|u(t)\|_{\beta} \leq C .
$$

Now, by Theorems 7.63 and 7.57 in [1] we get

$$
L^{2 \beta, q}(\Omega) \hookrightarrow W^{2 \beta-\varepsilon, q}(\Omega) \hookrightarrow C^{1}(\bar{\Omega}),
$$

for $N<(2 \beta-\varepsilon-1) q$. It suffices to take $q$ large. Similarly, we can reason with the equation for $v$. Hence, $\pi$ is compact and the proof is completed.

We are now ready to prove our main result.

Proof of Theorem 3.1. Once we have proved that $\pi$ is dissipative and compact, we need to build a continuous function $P$ verifying the hypotheses of Theorem 3.2. It is clear that

$$
S=\{(u, v) \in X: u=0 \quad \text { in } \Omega, \text { or } \quad v=0 \text { in } \Omega\},
$$

and so

$$
\omega(S)=\left\{(0,0),\left(0, \theta_{\mu}\right)\right\}
$$

Now, denote by $\varphi_{1}$ a positive eigenfunction associated to $\lambda_{1}$ and $\varphi_{\lambda}$ a positive eigenfunction associated $m(\lambda)=\lambda_{1}\left(-\Delta-\lambda \mathcal{G}\left(\theta_{\mu}\right)\right)$, that is

$$
-\Delta \varphi_{\lambda}-\lambda \mathcal{G}\left(\theta_{\mu}\right) \varphi_{\lambda}=m(\lambda) \varphi_{\lambda} \quad \text { in } \Omega, \quad \varphi_{\lambda}=0 \quad \text { on } \partial \Omega .
$$

Recall that $m(\lambda)<0$ for $\lambda>\lambda_{0}(\mu)$. Define

$$
P\left(u_{0}, v_{0}\right):=\left(\int_{\Omega} \varphi_{\lambda} u_{0} d x\right)^{\beta_{1}}\left(\int_{\Omega} \varphi_{1} v_{0} d x\right)^{\beta_{2}}
$$

with $\beta_{1}$ and $\beta_{2}$ positive constants to be chosen.

First, observe that $P$ can be written as

$$
P\left(u_{0}, v_{0}\right)=\exp \left\{\beta_{1} \log \left(\int_{\Omega} \varphi_{\lambda} u_{0} d x\right)+\beta_{2} \log \left(\int_{\Omega} \varphi_{1} v_{0} d x\right)\right\} .
$$

Take $\left(u_{0}, v_{0}\right)$ and denote by $\left(z_{1}, z_{2}\right)=\pi\left(u_{0}, v_{0}, t\right)$. Then,

$$
\begin{gathered}
\frac{P\left(z_{1}, z_{2}\right)}{P\left(u_{0}, v_{0}\right)}=\exp \left\{\beta_{1}\left[\log \left(\int_{\Omega} \varphi_{\lambda} z_{1}\right)-\log \left(\int_{\Omega} \varphi_{\lambda} u_{0}\right)\right]\right. \\
\left.+\beta_{2}\left[\log \left(\int_{\Omega} \varphi_{1} z_{2}\right)-\log \left(\int_{\Omega} \varphi_{1} v_{0}\right)\right]\right\} .
\end{gathered}
$$


Using that $\log (f(t))-\log (f(0))=\int_{0}^{t} f^{\prime}(s) / f(s) d s$ for a positive and regular function $f$, we get

$$
\begin{gathered}
\frac{P\left(z_{1}, z_{2}\right)}{P\left(u_{0}, v_{0}\right)}=\exp \left\{\beta_{1} \int_{0}^{t}\left[\frac{\int_{\Omega} \varphi_{\lambda}\left(\Delta z_{1}+\lambda z_{1}\left(1-z_{1}\right) \mathcal{G}\left(z_{2}\right)\right)}{\int_{\Omega} \varphi_{\lambda} z_{1}(x, s) d x}\right] d s+\right. \\
\left.\beta_{2} \int_{0}^{t}\left[\frac{\int_{\Omega} \varphi_{1}\left(\Delta z_{2}+\mu z_{2}-z_{2}^{2}-c z_{1} z_{2}\right)}{\int_{\Omega} \varphi_{1} z_{2}(x, s) d x}\right] d s\right\} .
\end{gathered}
$$

Using that

$$
\int_{\Omega} \varphi_{\lambda} \Delta z_{1}=\int_{\Omega}\left(-\lambda \mathcal{G}\left(\theta_{\mu}\right)-m(\lambda)\right) \varphi_{\lambda} z_{1}, \quad \text { and } \quad \int_{\Omega} \varphi_{1} \Delta z_{2}=-\lambda_{1} \int_{\Omega} \varphi_{1} z_{2},
$$

we get that

$$
\begin{aligned}
\frac{P\left(z_{1}, z_{2}\right)}{P\left(u_{0}, v_{0}\right)}= & \exp \left\{\beta_{1} \int_{0}^{t}\left[\frac{\int_{\Omega} \varphi_{\lambda} z_{1}\left(\lambda\left(1-z_{1}\right) \mathcal{G}\left(z_{2}\right)-\lambda \mathcal{G}\left(\theta_{\mu}\right)-m(\lambda)\right)}{\int_{\Omega} \varphi_{\lambda} z_{1}(x, s) d x}\right] d s+\right. \\
& \left.+\beta_{2} \int_{0}^{t}\left[\frac{\int_{\Omega} \varphi_{1} z_{2}\left(\mu-\lambda_{1}-z_{2}-c z_{1}\right)}{\int_{\Omega} \varphi_{1} z_{2}(x, s) d x}\right] d s\right\}
\end{aligned}
$$

Now, since $z_{1}$ and $z_{2}$ are bounded, there exist $d_{1}, d_{2} \in \mathbb{R}$ such that

$$
\lambda\left(1-z_{1}\right) \mathcal{G}\left(z_{2}\right)-\lambda \mathcal{G}\left(\theta_{\mu}\right)-m(\lambda) \geq d_{1}, \quad \mu-\lambda_{1}-z_{2}-c z_{1} \geq d_{2},
$$

hence

$$
\frac{P\left(z_{1}, z_{2}\right)}{P\left(u_{0}, v_{0}\right)} \geq \exp \left\{\left(\beta_{1} d_{1}+\beta_{2} d_{2}\right) t\right\}
$$

and so $a(t,(u, v))>0$ for some $t$.

It remains to show that $a(t,(u, v))>1$ for some $t>0$ if $(u, v) \in \omega(S)$. Take $(u, v) \in \omega(S)$ and $\left(u_{0}, v_{0}\right) \rightarrow(u, v)$. We distinguish two cases:

Case 1: $(u, v)=\left(0, \theta_{\mu}\right)$. Observe that if $\left(u_{0}, v_{0}\right)$ is near to $\left(0, \theta_{\mu}\right)$, then the solution $\left(z_{1}(x, t), z_{2}(x, t)\right)$ is near to $\left(0, \theta_{\mu}\right)$ for $t \in[0,1]$ for instance, because $\left(0, \theta_{\mu}\right)$ is the solution of $(1.2)$ with initial data $\left(0, \theta_{\mu}\right)$. Taking into account that multiplying the equation of $\theta_{\mu}$ by $\varphi_{1}$ we get,

$$
\int_{\Omega} \varphi_{1} \theta_{\mu}\left(\mu-\lambda_{1}-\theta_{\mu}\right)=0
$$

we conclude that for all $s \in[0,1]$

$$
\frac{\int_{\Omega} \varphi_{1} z_{2}\left(\mu-\lambda_{1}-z_{2}-c z_{1}\right)}{\int_{\Omega} \varphi_{1} z_{2}(x, s) d x} \rightarrow \frac{\int_{\Omega} \varphi_{1} \theta_{\mu}\left(\mu-\lambda_{1}-\theta_{\mu}\right)}{\int_{\Omega} \varphi_{1} \theta_{\mu} d x}=0, \quad \text { as }\left(u_{0}, v_{0}\right) \rightarrow\left(0, \theta_{\mu}\right) .
$$

Moreover, since $\left(z_{1}, z_{2}\right) \rightarrow\left(0, \theta_{\mu}\right)$ as $\left(u_{0}, v_{0}\right) \rightarrow\left(0, \theta_{\mu}\right)$ and $m(\lambda)<0$, for all $\varepsilon>0$ small enough we get

$$
\lambda\left(1-z_{1}\right) \mathcal{G}\left(z_{2}\right)-\lambda \mathcal{G}\left(\theta_{\mu}\right)-m(\lambda) \geq-\varepsilon-m(\lambda)>0,
$$


and so, using (3.7)

$$
a\left(1,\left(0, \theta_{\mu}\right)\right) \geq \exp \left(\beta_{1}(-\varepsilon-m(\lambda))\right)>1 .
$$

Case 2: $(u, v)=(0,0)$. In this case, if $\left(u_{0}, v_{0}\right) \rightarrow(0,0)$ then $\left(z_{1}, z_{2}\right) \rightarrow(0,0)$. Then, there exists $d \in \mathbb{R}$ such that

$$
\lambda\left(1-z_{1}\right) \mathcal{G}\left(z_{2}\right)-\lambda \mathcal{G}\left(\theta_{\mu}\right)-m(\lambda) \geq d-m(\lambda), \quad \mu-\lambda_{1}-z_{2}-c z_{1} \geq \frac{\mu-\lambda_{1}}{2}>0,
$$

and so,

$$
a(1,(0,0)) \geq \exp \left\{\beta_{1}(d-m(\lambda))+\beta_{2}\left(\frac{\mu-\lambda_{1}}{2}\right)\right\}>0
$$

taking $\beta_{2}$ large enough.

This proves the claim and completes the proof.

\section{Concluding remarks}

We have framed a simple model to study the difficulties that appear when the proliferation of EC only occurs when the TAF level reaches some threshold. We can summarize the behavior of the solutions of this model in the following manner.

Fix $\lambda>0$, i.e. the maximum mitotic rate of EC. We can calculate $\mu_{0}$ such that $\lambda_{0}\left(\mu_{0}\right)=\lambda$; it results $\lambda_{1}<\mu^{*}<\mu_{0}$ (see Figure 1 ). Then:

1. If $\mu \leq \lambda_{1}$, the growth rate of TAF is small. The consumption of TAF by EC and the decay of chemical lead the system to solution $v=0$ and so $u=0$ also. There is no angiogenesis.

2. If $\lambda_{1}<\mu<\mu_{0}$, then while TAF remains it is not sufficiently large for the proliferation of EC. The system tends to $\left(0, \theta_{\mu}\right)$. Again, there is no angiogenesis.

3. If $\mu>\mu_{0}$, the system is permanent, i.e., the solutions of the system maintain a distance from zero. In this case, the angiogenesis occurs.

Moreover, by Proposition 2.1 we know the behaviour of $\mu_{0}$ with respect to $\lambda$. In particular, if $\lambda$ increases to $+\infty$, then $\mu_{0} \rightarrow \mu^{*}$; while if $\lambda$ decreases to zero, then $\mu_{0} \rightarrow+\infty$.

\section{References}

[1] R. Adams, Sobolev spaces, Academic Press, New York, 1975.

[2] W. Allegretto, H. Xie, and S. Yang, Properties of solutions for a chemotaxis system, J. Math. Biol 35 (1997), 949-966.

[3] J. Blat, and K. J. Brown, Bifurcation of steady-state solutions in predator-prey and competition systems, Proc. R. Soc. Edinb. 97A (1984), 21-34. 
[4] H. Brezis, and L. Oswald, Remarks on sublinear elliptic equations, Nonlinear Anal. 10 (1986), 55-64.

[5] R. S. Cantrell, and C. Cosner, Spatial ecology via reaction-diffusion equations, Wiley Series in Mathematical and Computational Biology. John Wiley \& Sons, Ltd., Chichester, 2003.

[6] R. S. Cantrell, C. Cosner, and V. Hutson, Permanence in ecological systems with spatial heterogeneity, Proc. Royal Soc. Edin. 123A (1993), 533-559.

[7] M. A. J. Chaplain, Avascular growth, angiogenesis and vascular growth in solid tumours: the mathematical modelling of the stages of tumor development, Math. Comput. Modelling 23 (1996), 47-87.

[8] K. Deimling, Nonlinear Functional Analysis, Springer, New York, 1985.

[9] J. M. Fraile, J. López-Gómez, and J. C. Sabina de Lis, On the global structure of the set of positive solutions of some semilinear elliptic boundary value problems. J. Differential Equations 123 (1995), 180-212.

[10] D. Henry, Theory of semilinear parabolic equations, Lect. Notes in Math. 840, Springer-Verlag, Berlin, 1980.

[11] V. Hutson, A theorem on average Lyapunov functions, Monatsch. Math. 98 (1984), 267-275.

[12] J. López-Gómez, Nonlinear eigenvalues and global bifurcation: Application to the search of positive solutions for general Lotka-Volterra reaction-diffusion systems with two species, Diff. Int. Eqns., 7 (1994), 1427-1452.

[13] N. V. Mantzaris, S. Webb, and H. G. Othmer, Mathematical modeling of tumor-induced angiogenesis. J. Math. Biol. 49 (2004), 111-187.

[14] C. V. Pao, Nonlinear parabolic and elliptic equations, Plenum, New York, 1992.

[15] G. T. Whyburn, Topological Analysis, Princeton, 1955. 